\title{
A IMPORTÂNCIA DA
}

ASSISTÊNCIA DE ENFERMAGEM

NA ATENÇÃO BÁSICA À SAÚDE

\section{Volume 1}

ORGANIZADORES:

Andressa Prates Sá/ Dayane Araújo Rocha Keilla Silva Santos/Ingrid Isabel de Andrade Nhayeno Cordeiro Dantas/ Vinícius Duarte Silva Fernanda Santos Landim/ Francielle Araujo Bispo Weidny Eduardo de Sousa Silva/ Cecília Rodrigues Lima Anna Christina dos Reis Santos/ Thais Pereira Silva 


\section{A IMPORTÂNCIA DA}

ASSISTÊNCIA DE ENFERMAGEM

NA ATENÇÃO BÁSICA À SAÚDE

\section{Volume 1}

ORGANIZADORES:

Andressa Prates Sá/ Dayane Araújo Rocha Keilla Silva Santos/Ingrid Isabel de Andrade Nhayeno Cordeiro Dantas/Vinícius Duarte Silva Fernanda Santos Landim/ Francielle Araujo Bispo Weidny Eduardo de Sousa Silva/ Cecília Rodrigues Lima Anna Christina dos Reis Santos/ Thais Pereira Silva 
A IMPORTÂNCIA DA ASSISTÊNCIA DE ENFERMAGEM NA ATENÇÃO BÁSICA À SAÚDE

Volume: 1

$1^{\text {a }}$ Edição

TRIUNFO - PE 


\section{Editor-Chefe}

Me. Daniel Luís Viana Cruz

\section{Organizadores}

Andressa Prates Sá

Dayane Araújo Rocha

Keilla Silva Santos

Ingrid Isabel de Andrade

Nhayeno Cordeiro Dantas

Vinícius Duarte Silva

Fernanda Santos Landim

Francielle Araujo Bispo

Weidny Eduardo de Sousa Silva

Cecília Rodrigues Lima

Anna Christina dos Reis Santos

Thais Pereira Silva

\section{Conselho Editorial}

Dr. Cássio Brancaleone

Dr. Marcelo Luiz Bezerra da Silva

Dra. Pauliana Valéria Machado Galvão

Dr. Plínio Pereira Gomes Júnior

Dr. Walter Santos Evangelista Júnior

Dr. Wendel José Teles Pontes

Editores De Área - Ciências Da Saúde

Dra. Camyla Rocha de Carvalho Guedine

Dra. Cristieli Sérgio de Menezes Oliveira

Dr. Leandro dos Santos

Dr. Hugo Barbosa do Nascimento

Dr. Marcio Luiz Lima Taga 
Dra. Pauliana Valéria Machado Galvão

\section{Assistente Editorial}

Thialla Larangeira Amorim

\section{Imagem de Capa}

Freepik

\section{Edição de Arte}

Vileide Vitória Larangeira Amorim

\section{Revisão}

Os autores

\section{(2) $\odot \Theta \Theta$}

Este trabalho está licenciado com uma Licença Creative Commons - Atribuição-NãoComercialSemDerivações 4.0 Internacional.

O conteúdo abordado nos artigos, seus dados em sua forma, correção e confiabilidade são de responsabilidade exclusiva dos autores. 


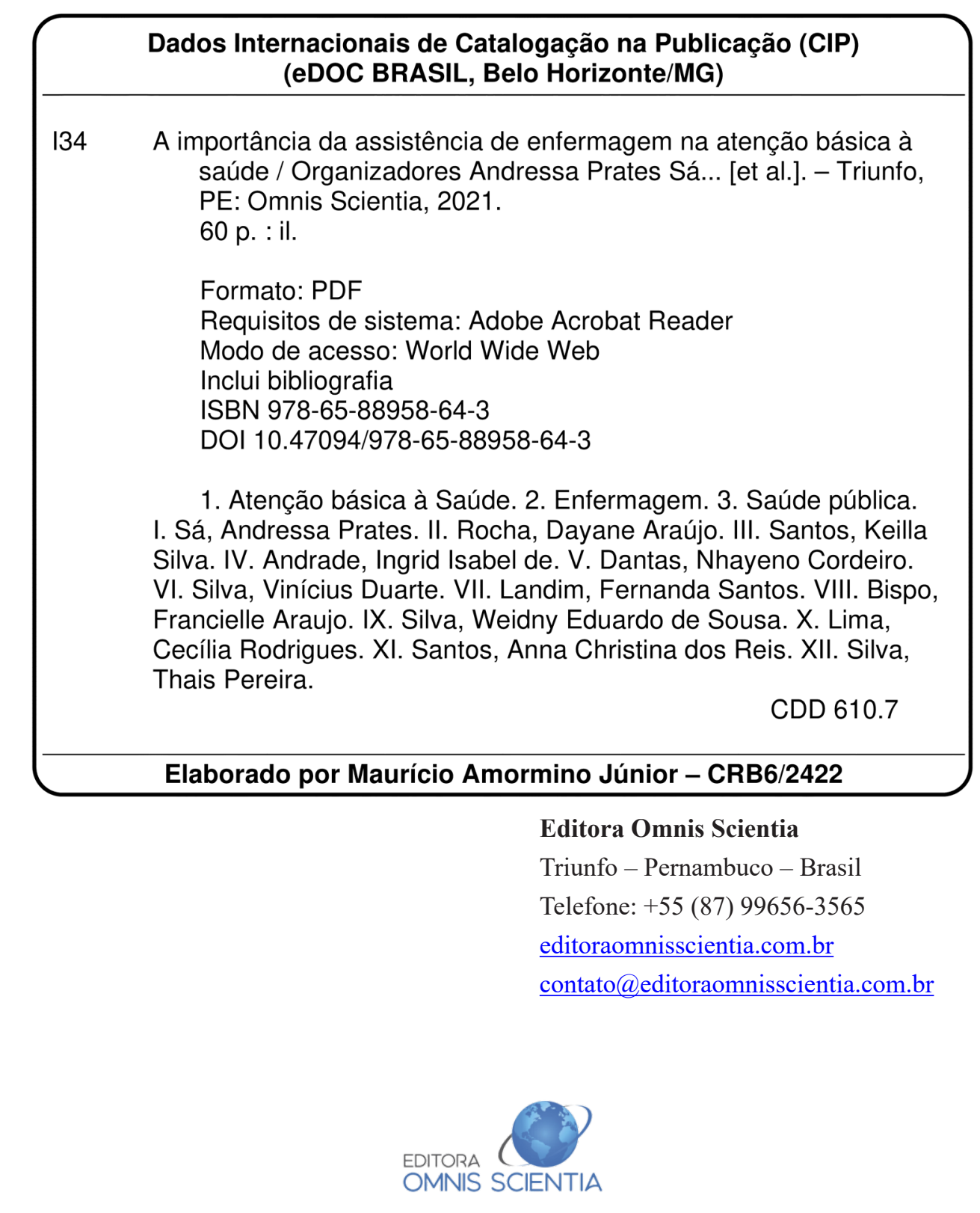




\section{PREFÁCIO}

O enfermeiro possui um papel fundamental dentro da atenção primária, pois nesse contexto esse profissional deve identificaros problemas desaúdee fatores de risco da população, monitoraras evoluções clínicas dos pacientes, participar e realizar ações voltadas para educação em saúde, realização e acompanhamento de tratamentos/reabilitação e a sistematização do cuidado dentro da atenção básica em todas as fases da vida humana, desde a gestação até a morte, dentro do cuidado integral do paciente, o atendendo no seu entorno biopsicossocial, assim concretizando várias das diretrizes do sistema único de saúde.

Este livro trás em seus capítulos estudos que evidenciam a necessidade e importância do trabalho desses profissionais da enfermagem dentro do sistema de saúde brasileiro, mostrando sua atuação dentro das diversas áreas, como na saúde da mulher, doenças crônicas e infectocontagiosas. 


\section{SUMÁRIO}

CAPÍTULO 1 . .12

A IMPORTÂNCIA DA ASSISTÊNCIA DE ENFERMAGEM AOS PACIENTES PORTADORES DE HANSENÍASE

Andressa Prates Sá

Dayane Araújo Rocha

Keilla Silva Santos

Ingrid Isabel de Andrade

Nhayeno Cordeiro Dantas

Vinícius Duarte Silva

Fernanda Santos Landim

Francielle Araujo Bispo

Weidny Eduardo de Sousa Silva

Cecília Rodrigues Lima

Aldair Almeida Batista

Thais Pereira Silva

DOI: 10.47094/978-65-88958-64-3/12-19

\section{CAPÍtULO 2.}

A RELEVÂNCIA DA EQUIPE DE ENFERMAGEM NA ASSISTÊNCIA DO CÂNCER DE COLO DO ÚTERO

Andressa Prates Sá

Dayane Araújo Rocha

Keilla Silva Santos

Ingrid Isabel de Andrade

Nhayeno Cordeiro Dantas

Vinícius Duarte Silva 
Fernanda Santos Landim

Francielle Araujo Bispo

Weidny Eduardo de Sousa Silva

Cecília Rodrigues Lima

Anna Christina dos Reis Santos

Thais Pereira Silva

DOI: 10.47094/978-65-88958-64-3/20-27

CAPÍTULO 3

A IMPORTÂNCIA DA ASSISTÊNCIA DA EQUIPE DE ENFERMAGEM AOS PACIENTES COM HIPERTENSÃO ARTERIAL SISTÊMICA

Andressa Prates Sá

Dayane Araújo Rocha

Keilla Silva Santos

Ingrid Isabel de Andrade

Nhayeno Cordeiro Dantas

Vinícius Duarte Silva

Fernanda Santos Landim

Francielle Araujo Bispo

Weidny Eduardo de Sousa Silva

Cecília Rodrigues Lima

Anna Christina dos Reis Santos

Thais Pereira Silva

DOI: 10.47094/978-65-88958-64-3/28-36 
ASSISTÊNCIA DOS PROFISSIONAL DE ENFERMAGEM DIANTE A MULHER COM O CÂNCER DE MAMA

Andressa Prates Sá

Dayane Araújo Rocha

Keilla Silva Santos

Ingrid Isabel de Andrade

Nhayeno Cordeiro Dantas

Vinícius Duarte Silva

Fernanda Santos Landim

Francielle Araujo Bispo

Weidny Eduardo de Sousa Silva

Cecília Rodrigues Lima

Anna Christina dos Reis Santos

Thais Pereira Silva

DOI: 10.47094/978-65-88958-64-3/37-46

CAPÍtULO 5.

A RELEVÂNCIA DA ASSISTÊNCIA DA EQUIPE DE ENFERMAGEM NA ORIENTAÇÃO DO ALEITAMENTO MATERNO

Andressa Prates Sá

Dayane Araújo Rocha

Keilla Silva Santos

Ingrid Isabel de Andrade

Nhayeno Cordeiro Dantas

Vinícius Duarte Silva

Fernanda Santos Landim

Francielle Araujo Bispo 
Weidny Eduardo de Sousa Silva

Cecília Rodrigues Lima

Anna Christina dos Reis Santos

Thais Pereira Silva

DOI: 10.47094/978-65-88958-64-3/47-57 


\title{
A IMPORTÂNCIA DA ASSISTÊNCIA DE ENFERMAGEM AOS PACIENTES PORTADORES DE HANSENÍASE
}

\author{
Andressa Prates Sá ${ }^{1}$ \\ Faculdade de Saúde e Humanidades Ibituruna - FASI, Montes Claros, Minas Gerais. \\ https://orcid.org/0000-0002-9892-7191
}

\section{Dayane Araújo Rocha ${ }^{2}$}

Enfermeira Graduada Universidade do Estado de Minas Gerais.

https://orcid.org/0000-0001-9594-3287

\section{Keilla Silva Santos ${ }^{3}$}

Faculdade de Saúde e Humanidades Ibituruna- FASI, Montes Claros, Minas Gerais.

http://lattes.cnpq.br/4390347860194905

\section{Ingrid Isabel de Andrade ${ }^{4}$}

Faculdade de Saúde e Humanidades Ibituruna - FASI, Montes Claros, Minas Gerais. http://lattes.cnpq.br/8559852008026622

\section{Nhayeno Cordeiro Dantas 5}

Faculdade de Saúde e Humanidades Ibituruna - FASI, Montes Claros, Minas Gerais. http://lattes.cnpq.br/5756148457135856

\section{Vinícius Duarte Silva ${ }^{6}$}

Faculdade de Saúde e Humanidades Ibituruna - FASI, Montes Claros, Minas Gerais. http://lattes.cnpq.br/0383688388186075

\section{Fernanda Santos Landim ${ }^{7}$}

Graduada em Enfermagem pela Universidade Estadual de Montes Claros.

http://lattes.cnpq.br/8867397116277703

\section{Francielle Araujo Bispo ${ }^{8}$}

Faculdade de Saúde e Humanidades Ibituruna - FASI, Montes Claros, Minas Gerais. http://lattes.cnpq.br/8128766871065494 


\title{
Weidny Eduardo de Sousa Silva9
}

Faculdade de Saúde e Humanidades Ibituruna - FASI, Montes Claros, Minas Gerais.

http://lattes.cnpq.br/5249560920697190

\section{Cecília Rodrigues Lima ${ }^{10}$}

Faculdade de Saúde e Humanidades Ibituruna - FASI, Montes Claros, Minas Gerais. http://lattes.cnpq.br/1190180224007265

\author{
Aldair Almeida Batista ${ }^{11}$ \\ Faculdades Unidas do Norte de Minas - FUNORTE \\ http://lattes.cnpq.br/1956076224184984

\section{Thais Pereira Silva ${ }^{12}$} \\ Faculdade de Saúde e Humanidades Ibituruna- FASI, Montes Claros, Minas Gerais. \\ $\underline{\text { http://lattes.cnpq.br/78744455243844985 }}$
}

RESUMO: Introdução: A hanseníase é uma doença infectocontagiosa crônica ocasionada pelo Mycobacterium leprae ou bacilo de Hansen, um parasita intracelular obrigatório com afinidade por células cutâneas e nervos periféricos. Objetivo: Este estudo fluiu com o objetivo de descrever a importância da assistência de enfermagem aos pacientes portadores de Hanseníase. Metodologia: Trata-se de um estudo de abordagem qualitativa, através da identificação de estudos sobre o tema a fim de descrever a importância da atuação da enfermagem na prevenção, diagnóstico e tratamento da Hanseníase. A seleção dos estudos foi realizada através de busca em bancos de dados online como: Scielo, google acadêmico, com base nos critérios: ano de publicação entre 2009 e 2021, publicados em português, espanhol e inglês e artigos com textos completos. Resultados e Discussão: Contudo intervir no processo de atenção à pessoa atingida pela hanseníase, tendo o propósito de prevenir incapacidades físicas e promover o autocuidado, incentivando em sua capacidade para o trabalho, vida social e aspectos psicológicos. Os enfermeiros identificaram que a dificuldade da hanseníase é quase impossível ao usuário não se curvar à força do estigma que aflige a normalidade do viver. Conclusão: O estudo é efetivo à medida que possibilita conhecer a ação da enfermagem no contexto domiciliar, enfatizando as atividades de autocuidado e a necessidade da educação em saúde, para o prognóstico positivo do paciente. Com o conhecimento adquirido na revisão integrativa, observa-se todas as dificuldades que são impostas a assistência de enfermagem aos pacientes no domicílio e enfatiza a participação da APS na educação dos portadores de hanseníase.

PALAVRAS-CHAVES: Hanseníase. Assistência de Enfermagem. Prevenção de Doença. 


\section{THE IMPORTANCE OF NURSING CARE FOR PATIENTS WITH LEPROSY}

ABSTRACT: Introduction: Leprosy is a chronic infectious disease caused by Mycobacterium leprae or Hansen's bacillus, an obligatory intracellular parasite with affinity for skin cells and peripheral nerves. Objective: This study aimed to describe the importance of nursing care for patients with leprosy. Methodology: This is a study with a qualitative approach, through the identification of studies on the subject in order to describe the importance of the role of nursing in the prevention, diagnosis and treatment of leprosy. The selection of studies was performed by searching online databases such as: Scielo, academic google, based on the criteria: year of publication between 2009 and 2021, published in Portuguese, Spanish and English and articles with full texts.Results and discussion: However, intervening in the process of care for people affected by leprosy, with the purpose of preventing physical disabilities and promoting self-care, encouraging their ability to work, social life and psychological aspects. The nurses identified that the difficulty of leprosy is almost impossible for the user not to bend to the force of the stigma that afflicts the normality of living. Conclusion: The study is effective insofar as it makes it possible to know the nursing action in the home context, emphasizing self-care activities and the need for health education for the positive prognosis of the patient. With the knowledge acquired in the integrative review, all the difficulties that are imposed on nursing care for patients at home are observed and it emphasizes the participation of PHC in the education of leprosy patients.

KEY-WORDS: Leprosy. Nursing Assistance. Disease prevention.

\section{INTRODUÇÃO}

A hanseníase é uma doença infectocontagiosa crônica ocasionada pelo Mycobacterium leprae ou bacilo de Hansen, um parasita intracelular obrigatório com afinidade por células cutâneas e nervos periféricos. A única fonte de infecção considerada da doença é o homem, e o principal meio de contágio é através de secreções nasais e gotículas do trato respiratório de pacientes não tratados, outro aspecto importante é que a hanseníase infecta muitas pessoas, entretanto, poucas adoecem, visto que o bacilo apresenta alta infectividade e baixa patogenicidade (SILVA et al., 2016).

Compreender que mesmo com os avanços ocorridos no diagnóstico, controle e tratamento da hanseníase, até então é nítido o estigma da doença, provocado pelo preconceito, receio e pelas rejeições por parte da sociedade. O enfermeiro é de suma importância no tratamento do paciente com hanseníase, esclarecendo quanto a importância do tratamento, sua eficácia e efeitos colaterais, assim como acompanhando-o durante todo o tratamento (DUARTE; AYRES; SIMONETTI, 2009).

Os principais aspectos do enfermeiro no acompanhamento do paciente com hanseníase, é a dose supervisionada, assegurando assim a continuidade do tratamento, realizar busca ativa de faltosos e de todos os contatos, identificando novos casos e encaminhando para confirmação diagnóstica, efetuar a dispensação de medicamentos e acompanhando sempre de perto em busca da cura e obtenção 
de alta do paciente (BENTO et al., 2016).

Tendo em consideração os aspectos epidemiológicas, a atuação da enfermagem nos casos de fatores que levam ao abandono do tratamento da Hanseníase, necessitar-se realizada pelo enfermeiro, e demais integrantes da equipe de enfermagem, de forma sistemática, por meio de gerenciamento da ingestão regular das doses farmacológicas utilizadas no tratamento, precisam ser supervisionadas, favorecendo também a conscientização dos portadores, familiares e comunidade em relação à gravidade do efeito multiplicador da contaminação (SILVA et al., 2016).

A prevenção de incapacidades, de acordo com o Ministério da Saúde (2010), começa por meio do diagnóstico precoce, tratamento com a PQT, reabilitação, exame dos contatos e aplicabilidade da vacina BCG (Bacilo de Calmette e Guérin) da mesma forma, tratamento apropriado das reações hansênicas e neurites, orientações na prevenção do autocuidado, bem como suporte emocional e social.

\section{OBJETIVO}

Com base nessas considerações, o presente estudo tem como objetivo descrever a importância da assistência de enfermagem aos pacientes portadores de Hanseníase.

\section{METODOLOGIA}

Trata-se de um estudo de abordagem qualitativa, através da identificação de estudos sobre o tema a fim de descrever a importância da atuação da enfermagem na prevenção, diagnóstico e tratamento da Hanseníase entre os anos de 2009 e 2021. Adotou-se a revisão integrativa da literatura devido a sua contribuição para a análise de resultados.

A revisão integrativa da literatura estabelece critérios para a coleta e análise de dados, desta forma adotamos seis etapas indicadas para a elaboração da revisão integrativa que são: a seleção da pergunta em que consiste a pesquisa; a definição dos critérios de inclusão dos estudos e seleção da amostra; a representação dos estudos selecionados em tabela, representado na tabela 1; análise crítica dos dados encontrados e a discussão dos resultados obtidos.

A seleção dos estudos foi realizada através de busca em bancos de dados online como: Scielo, google acadêmico, com base nos critérios: ano de publicação entre 2009 e 2021; publicados em português, espanhol e inglês e artigos com textos completos.

Após a leitura dos oito artigos, todos foram incluídos para análise, pois os artigos apresentaram aspectos relacionados à importância da enfermagem no acompanhamento de pacientes de hanseníase. Foram utilizados 8 artigos, e referenciados no presente texto descritos na tabela 1. 


\section{RESULTADOS E DISCUSSÃO}

Tabela 1: Descrição dos artigos quanto ao instrumento de avaliação e seus resultados.

\begin{tabular}{|c|c|c|}
\hline AUTORES & OBJETIVO & TIPO DE ESTUDO \\
\hline SILVA et al., 2016. & $\begin{array}{l}\text { Analisar a assistência de enfermagem } \\
\text { utilizada no atendimento de portadores } \\
\text { de hanseníase. }\end{array}$ & $\begin{array}{c}\text { Estudo retrospectivo, prospectivo, } \\
\text { com abordagem quantitativa. }\end{array}$ \\
\hline BENTO et al., 2016. & $\begin{array}{l}\text { Compreender o papel da enferma- } \\
\text { gem no acompanhamento de casos } \\
\text { de hanseníase no Brasil, por meio da } \\
\text { literatura. }\end{array}$ & $\begin{array}{c}\text { Uma revisão integrativa da litera- } \\
\text { tura. }\end{array}$ \\
\hline PASCHOAL; SOLER, 2014. & $\begin{array}{l}\text { Descrever alguns fatos acerca do papel } \\
\text { do enfermeiro no cuidado aos pacien- } \\
\text { tes com hanseníase e seu relevante e } \\
\text { necessário envolvimento com as ações } \\
\text { de controle da doença. }\end{array}$ & Estudo descritivo. \\
\hline $\begin{array}{c}\text { DUARTE; AYRES; SIMONET- } \\
\text { TI, } 2009 .\end{array}$ & $\begin{array}{l}\text { Analisar o instrumento de consulta de } \\
\text { enfermagem utilizado junto à clientela } \\
\text { atendida no Programa de Hanseníase } \\
\text { de uma Unidade de Atenção Primária } \\
\text { à Saúde e identificar as principais } \\
\text { necessidades de saúde e as ações de } \\
\text { enfermagem propostas. }\end{array}$ & Estudo descritivo. \\
\hline $\begin{array}{c}\text { VIANA; AGUIAR; AQUINO, } \\
2016 .\end{array}$ & $\begin{array}{l}\text { Caracterizar a população idosa afetada } \\
\text { por hanseníase quanto aos aspectos } \\
\text { sócio epidemiológicos e clínicos. }\end{array}$ & $\begin{array}{l}\text { Trata-se de um estudo descritivo, } \\
\text { com abordagem quantitativa. }\end{array}$ \\
\hline $\begin{array}{l}\text { BEZERRA; NUNES; COSTA } \\
\text { DE JESUS, } 2019 .\end{array}$ & $\begin{array}{l}\text { Analisar o perfil diagnóstico de usuá- } \\
\text { rios acometidos pela hanseníase, le- } \\
\text { vantando diagnósticos de enfermagem, } \\
\text { com foco no problema conforme a ta- } \\
\text { xonomia II da NANDA-I 2015-2017, } \\
\text { à luz do construto do autocuidado } \\
\text { de Orem. }\end{array}$ & $\begin{array}{l}\text { Um estudo descritivo, transversal de } \\
\text { abordagem quantitativa. }\end{array}$ \\
\hline SILVA; PAZ, 2017. & $\begin{array}{l}\text { Analisar a prática de enfermeiros na } \\
\text { experiência de cuidar de pessoas com } \\
\text { hanseníase em serviços de saúde do } \\
\text { município do Rio de Janeiro. }\end{array}$ & Estudo qualitativo. \\
\hline SANTOS et al., 2021. & $\begin{array}{l}\text { Identificar na literatura as principais } \\
\text { ações desenvolvidas pela equipe de } \\
\text { enfermagem no âmbito domiciliar, } \\
\text { para ofertar qualidade de vida e me- } \\
\text { lhorar o prognóstico da pessoa porta- } \\
\text { dora de hanseníase, apontando a im- } \\
\text { portância de praticar o autocuidado. }\end{array}$ & $\begin{array}{l}\text { Estudo do tipo revisão integrativa de } \\
\text { aspecto qualitativo. }\end{array}$ \\
\hline
\end{tabular}

Fonte: Próprios autores. Uma revisão integrativa da literatura. 
Contudo o paciente acometido pela hanseníase, procura como atendimento inicial a atenção primária à saúde, nesta situação sucede o atendimento feito pela equipe multiprofissional, em essencial às ações de enfermagem, deste modo caracteriza assim como, o cuidado contínuo ou vínculo de longitudinalidade, o que constata com a conduta desenvolvida no domicílio (SANTOS et al., 2021).

Achados na literatura trazem a evidência do diagnóstico de enfermagem tendo conhecimento deficiente ocorrente e relevante para as discussões em indivíduos e grupos específicos que têm o nível de conhecimento como subsídio para a adesão ao tratamento e continuidade do mesmo, bem como, para retomar a responsabilidade que o enfermeiro tem de discorrer ao paciente a ciência sobre o devido acometido patológico ou afecções que o cerca (BEZERRA; NUNES; COSTA DE JESUS, 2019).

Segundo os estudos obtidos por Viana, Aguiar e Aquino (2016),o enfermeiro atua além da consulta de enfermagem, na realização de exames, avaliações, e encaminhamentos na procura precoce dos achados no exame dermatoneurológico para corrigir rapidamente as causas, na prevenção de infecções oportunistas na época do tratamento, em decorrência da inibição da resistência, no envio de materiais para exames laboratoriais, na formação de grupos de educação em saúde e, na assistência para pacientes com ou sem problemas relacionados aos estados reacionais para a solução, encaminhamento ou nas orientações e problemática que as reações possam gerar no paciente.

Através de um estudo conduzido por Viana, Aguiar e Aquino (2016), as incapacidades podem ser acompanhadas de dor intensa, insensibilidade do nervo, edema, déficit motor e sensitivo ou pode se desenvolver sem dor, caracterizando as neurites silenciosas, as quais não dispõem os achados de dor ou hipersensibilidade do nervo, as alterações de sensibilidade e/ou de força motora são identificadas através de exame físico direcionado, o que torna essencial na avaliações periódicas, mesmo na ausência de queixas.

Contudo intervir no processo de atenção à pessoa atingida pela hanseníase, tendo o propósito de prevenir incapacidades físicas e promover o autocuidado, incentivando em sua capacidade para o trabalho, vida social e aspectos psicológicos (BENTO et al., 2016).

O papel do enfermeiro no cuidado aos pacientes com hanseníase em crise reacional, especialmente na detecção precoce da neurite silenciosa com a palpação continuada dos nervos periféricos, na avaliação neurológica simplificada intencionando a prevenção de incapacidades, na observação da mudança de coloração e aspecto das manchas e na detecção de edemas de extremidades (PASCHOAL; SOLER, 2014)

Todas as adversidades encontradas no domicílio, a não adesão ao regime medicamentoso é que se encontra com maior frequência, e por conseguinte obteve-se também os problemas relacionados à percepção sensorial, relacionada principalmente ao acometimento dos nervos periféricos, sendo a assistência de enfermagem o cuidado que busca mais à frente de reconhecer as principais causas de complicações, e também fatores que atuam indiretamente com o prognóstico positivo, enfatizando especialmente os hábitos saudáveis de vida (SANTOS et al., 2021). 
De acordo com os estudos de Silva e Paz (2017), os enfermeiros identificaram que a dificuldade da hanseníase é quase impossível ao usuário não se curvar à força do estigma que aflige a normalidade do viver. A fragilidade das informações a respeito da patologia que pode acarretar prejuízos individuais e sociais, por essa razão costuma orientar o usuário, os familiares e os profissionais de saúde. Semelhante aos resultados deste estudo, investigação realizada em município mineiro apresentou que as reações dos doentes foram amenizadas pelo acolhimento e informação.

\section{CONCLUSÃO}

Um dos limites deste estudo, foi a pouca quantidade de artigos na íntegra, que abordassem com objetividade a importância da assistência de enfermagem aos pacientes portador de hanseníase, enfatizando o autocuidado e assim foi necessário a elaboração de novos estudos que abordem enfaticamente intervenções desses profissionais neste contexto.

O estudo é efetivo à medida que possibilita conhecer a ação da enfermagem no contexto domiciliar, enfatizando as atividades de autocuidado e a necessidade da educação em saúde, para o prognóstico positivo do paciente. Com o conhecimento adquirido na revisão integrativa, observa-se todas as dificuldades que são impostas a assistência de enfermagem aos pacientes no domicílio e enfatiza a participação da APS na educação dos portadores de hanseníase.

Envidar-se a necessidade da promoção de saúde em todos os eixos do cuidado, em especial práticas voltadas à atenção aos pacientes portadores de hanseníase, e a capacitação da equipe de saúde para exercer as atividades com efetividade. Frente a problemática apontada no referido estudo, aponta-se a necessidade do desenvolvimento de novos estudos voltados à temática da assistência de enfermagem ao paciente portador de hanseníase.

Portanto as ações através de organização, gestão e administração do programa de controle da hanseníase fazem parte também das atribuições do enfermeiro na sua rotina diária de atendimento. Assim sendo, a contribuição para ter êxito na obtenção da cura, e a prevenção dos comunicantes, precisam de informações e ações educativas, na perspectiva de inserir saberes clínicos, nas relações dos pacientes com seu tratamento, com o objetivo de obter adesão ao tratamento realizado.

\section{DECLARAÇÃO DE INTERESSES}

Nós, autores deste capítulo, declaramos que não possuímos conflitos de interesses de ordem financeira, comercial, política, acadêmica e pessoal.

\section{REFERÊNCIA}

SILVA, Liniker Scolfild Rodrigues da et al. A assistência de enfermagem aos portadores de hanseníase assistidos pelo programa de saúde da família. Rev. enferm. UFPE on line, p. 4111-4117, 2016. 
DUARTE, Marli Teresinha Cassamassimo; AYRES, Jairo Aparecido; SIMONETTI, Janete Pessuto. Consulta de enfermagem: estratégia de cuidado ao portador de hanseníase em atenção primária. Texto \& Contexto-Enfermagem, v. 18, p. 100-107, 2009.

BENTO, D. A. B.; SOUSA, M. N. A. de; PEREIRA G. da S. A.; TEMOTEO R. C. de A. Acompanhamento da hanseníase no Brasil: O papel da enfermagem. Faculdades Integradas de Patos. Rev. Journal of Medicine and health Promotion, 1(1):166-179, 2016.

BRASIL, Ministério da Saúde. Portaria no 594 de 29 de outubro de 2010b. Inclui Cadastro Nacional de Estabelecimentos de Saúde, o serviço de Atenção Integral em Hanseníase. Diário Oficial da União. Brasília, DF, 04 nov 2010. Disponível em:. Acesso em: nov. 2019.

PASCHOAL, Vania Del'Arco; SOLER, Zaida Aurora Sperli Geraldes. O fenômeno reacional na hanseníase e aspectos da assistência de enfermagem. Revista Família, Ciclos de Vida e Saúde no Contexto Social, v. 3, n. 1, 2015.

SILVA, Maria Cristina Dias da; PAZ, Elisabete Pimenta Araújo. Experiências de cuidado dos enfermeiros às pessoas com hanseníase: contribuições da hermenêutica. Acta Paulista de Enfermagem, v. 30, p. 435-441, 2017.

DE SOUSA SANTOS, Allan Bruno Alves et al. Assistência de enfermagem domiciliar ao portador de hanseníase: Um incentivo ao autocuidado. Research, Society and Development, v. 10, n. 9, p. e16810918041-e16810918041, 2021.

BEZERRA, Maria Luiza Rêgo. Diagnósticos de enfermagem com foco no problema para indivíduos acometidos pela hanseníase. Revista Enfermagem Atual In Derme, v. 88, n. 26, 2019.

VIANA, Lucian da Silva; AGUIAR, Maria Isis Freire de; AQUINO, Dorlene Maria Cardoso de. Perfil sócio epidemiológico e clínico de idosos afetados por hanseníase: contribuições para a enfermagem, 2016. 


\section{ÍNDICE REMISSIVO}

$\mathbf{A}$

ação da enfermagem no contexto domiciliar 13, 18

agentes patogênicos 48,50

aleitamento materno $48,49,50,51,52,53,54,55,56,57$

alimentos pré-lácteos 48,50

alterações metabólicas 29, 30

amamentação $48,49,50,51,52,53,54,55,56,57$

assistência de enfermagem 13, 15, 16, 17, 18, 19, 24, 26, 29, 32, 38, 43, 44, 45, 46, 52, 53, 54, 55, 56,57

assistência de enfermagem aos portadores de Hipertensão Arterial Sistêmica 29

atividades de autocuidado 13,18

atuação da enfermagem na prevenção, diagnóstico e tratamento da Hanseníase 13, 15

B

bacilo de Hansen 13, 14

benefícios do aleitamento materno 48

C

câncer $21,22,23,24,25,26,33,38,39,40,41,42,43,44,45,46$

câncer cérvico uterino 21,22

câncer de colo de útero 21, 23, 24, 25, 26

carcinoma de útero 21,25

colostro 48,50

D

deserção do tabagismo 29, 31

dificuldade da hanseníase 13

dificuldades vivenciados pelas mães 48,53

doença circulatória 29, 30

doença infectocontagiosa crônica 13, 14

doenças cardiovasculares fatais e não fatais 29,30

$\mathbf{E}$

educação dos portadores de hanseníase 13

educação em saúde 13, 17, 18, 24, 26, 33, 34, 36, 48, 51, 53, 55

equipe de enfermagem na assistência do câncer de colo do útero 21, 23, 24

equipe de enfermagem na orientação do aleitamento materno 48 
exame citopatológico 21, 23

exercícios físicos $25,29,31,34$

G

gestantes $21,23,50,51,52,53$

H

hábitos de vida 29, 31, 33, 38, 40

hanseníase 13, 14, 15, 16, 17, 18, 19

Hipertensão Arterial 29, 30, 31, 32, 35

hipotermia 48, 50

histerectomia parcial 21, 23

I

incapacidades físicas 13,17

$\mathbf{L}$

lesões no intestino imaturo 48, 50

M

mamografia 38, 40, 44

maturação do epitélio intestinal 48,50

menopausa 21, 23

mulheres com câncer de mama 38, 41

Mycobacterium leprae 13, 14

$\mathbf{N}$

neoplasia $38,39,44$

neoplasia mamária 38, 44

$\mathbf{P}$

parasita intracelular obrigatório 13, 14

perda do controle da divisão celular 21,22

portadores de Hanseníase 13, 15

prática da amamentação 48

processo de adoecimento 38,41

Q

qualidade de vida $16,25,31,34,35,48,56$

$\mathbf{R}$

recém-nascido $48,49,50,52,53,56$ 
saúde pública 29, 34, 38, 39, 44, 50

T

tratamento do câncer de colo de útero 21, 25

V

vida sexual 21,23

vida sexual ativa 21,23

virgens 21,23 
editoraomnisscientia@gmail.com M https://editoraomnisscientia.com.br/ $\circledast$

@editora_omnis_scientia (0) https://www.facebook.com/omnis.scientia.9 f

$$
\text { +55 (87) 9656-3565 }
$$


editoraomnisscientia@gmail.com M https://editoraomnisscientia.com.br/ $\circledast$

@editora_omnis_scientia (0) https://www.facebook.com/omnis.scientia.9 f

$$
\text { +55 (87) 9656-3565 }
$$

